\title{
Survey of incidence of diverticular disease, dietary advice and probiotic advice in three Surrey practices
}

\author{
J. A. A. Nichols ${ }^{1}$ and L. Thomas ${ }^{2}$ \\ ${ }^{1} 60$ Manor Way, Onslow Village, Guildford, Surrey GU2 7RR, UK and ${ }^{2}$ Yakult UK Ltd, Artemis, Odyssey Business Park, \\ West End Road, South Ruislip, Middlesex HA4 6QE, UK
}

Diverticulosis is a deficiency disease caused by a shortage of dietary fibre ${ }^{(1)} ; 20 \%$ of subjects will develop diverticulitis and need antibiotics $^{(2)}$. Dietary advice includes increasing intake of vegetable fibre, but giving probiotics during and after antibiotics for a diverticulitis attack may also be beneficial ${ }^{(3,4)}$. Estimates of the prevalence of diverticulosis based on subjects aged $\geq 40$ years vary from $6-8 \%$ in African countries to $25-50 \%$ in European countries ${ }^{(5,6)}$. In North America the incidence in subjects aged $>50$ years has been estimated at $40 \%$ and $10-25 \%$ developed diverticulitis ${ }^{(2)}$. According to these studies there is no significant gender bias. However, in the present survey, data derived from UK primary-care electronic records show a lower prevalence of diverticulosis of $11 \%$ and the attack rate for diverticulitis per 5 years is two to three times higher in females than males $(P<0.001)$. Although there are more women than men with known diverticulosis, female longevity is a confounding factor. The $7.4 \%$ prevalence of diverticulosis in subjects aged $>55$ years from the general practitioner (GP) records may be an underestimate compared with total population screening for diverticulosis ${ }^{(5,6)}$.

Results of a questionnaire sent out to patients who had been treated for diverticulitis in the previous 5 years indicated that $\geq 31 \%$ of patients with diverticulitis retained GP advice on dietary fibre and $15.6 \%$ recalled being advised to take a probiotic. At the time of completion of the questionnaire $32.5 \%$ of subjects were taking a probiotic regularly and further data analysis showed a trend for these subjects to have fewer bowel symptoms and slightly fewer episodes of diverticulitis, which did not reach significance. Several responses to an open-ended comments section seemed to back up this trend: 'Since starting to take a liquid probiotic daily (friend recommendation) frequency \& discomfort of attacks has reduced'.

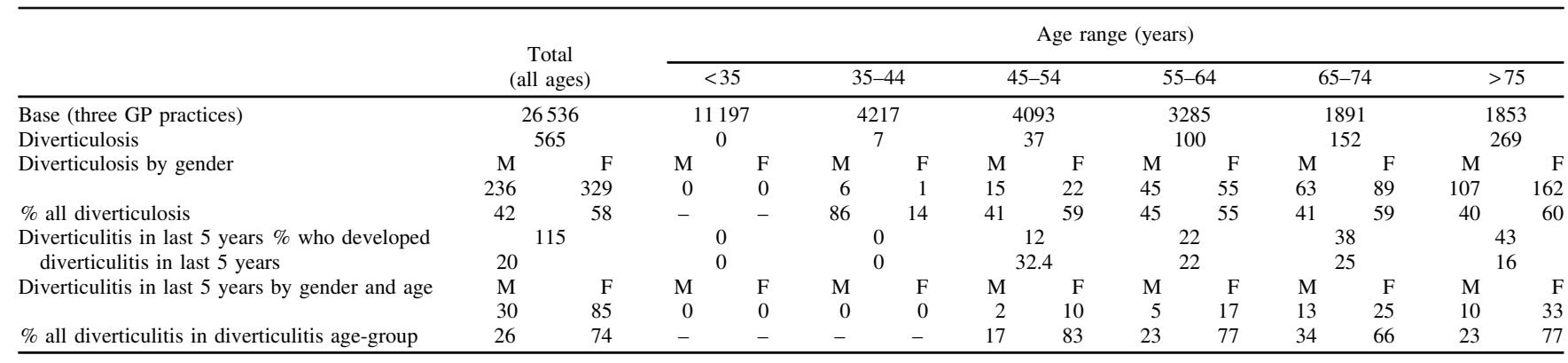

M, male; F, female.

Patients who stay on a long-term daily probiotic regimen appear to have fewer attacks of diverticulitis but the power of this retrospective survey was inadequate to test the hypothesis that probiotics are genuinely beneficial. A larger prospective trial is needed and it is suggested that subjects should be recruited who have had two or more episodes of diverticulitis. An open label pilot study of a daily dose of a probiotic for 2 years could be the next stage but ultimately a randomised control trial of probiotic $v$. a placebo will be essential.

1. Santhini J \& Savvas P (2008) Int J Colorectal Dis 23, 619-627.

2. Parks TG (1975) Clin Gastroenterol 4, 53-69.

3. Giaccari S, Tronci S, Falconieri M et al. (1993) Riv Eur Sci Med Farmacol 15, 29-34.

4. Fric P \& Zavarol M (2003) Eur J Gastroenterol Hepatol 15, 313-315.

5. Blachut K, Paradowski L \& Garcarek J (2004) Rom J Gastroenterol 13, 281-285.

6. Hughes LE (1969) Gut 10, 336-351. 